\title{
Tromboembolismo pulmonar: estratificación de riesgo y dilemas terapéuticos
}

'Departamento de

Medicina Interna.

${ }^{2}$ Departamento de

Medicina Intensiva.

Facultad de Medicina,

Pontificia Universidad

Católica de Chile. Santiago

de Chile.

Recibido el 29 de diciembre de 2011, aceptado el 9 de mayo de 2012.

Correspondencia a:

Dr. Max Andresen $\mathrm{H}$.

Departamento de

Medicina Intensiva

Facultad de Medicina PUC

Marcoleta $367,4^{\circ}$ piso,

Santiago,Chile

E-mail: andresen@med.

puc.cl

\author{
ANDRÉS AIZMAN ${ }^{1}$, MARCELO MERCADO $^{2}$, MAX ANDRESEN $^{2}$
}

\section{Risk stratification of pulmonary thromboembolism}

The prognosis of pulmonary thromboembolism (PE) is related to the cardiopulmonary reserve of the patient and the magnitude of the embolus that impacts pulmonary circulation. The presence of hemodynamic instability (shock) stratifies a group of patients with high mortality, which should be treated with thrombolysis. Patients without shock but with right ventricular dysfunction can have a dismal evolution and should be managed aggressively. CAT scan, echocardiography and serum markers can be of value to define patients with a higher mortality. The available evidence to define the best diagnostic and therapeutic strategy is scanty, controversial and inconclusive. A good combination of clinical, imaging and biological markers should be defined to identify those patients without shock but with a high rate of complications and mortality, that could benefit from aggressive treatments.

(Rev Med Chile 2012; 140: 1482-1489).

Key words: Shock, cardiogenic; Thrombolysis, therapeutic; Venous thromboembolism.

$\mathrm{E}$ 1 tromboembolismo pulmonar (TEP) constituye, en frecuencia, la tercera patología cardiovascular después del infarto agudo al miocardio y el accidente cerebro-vascular ${ }^{1}$.

A pesar de los avances en prevención, técnicas diagnósticas y opciones terapéuticas, el TEP sigue siendo una patología frecuentemente subdiagnosticada y potencialmente letal. Así, series de autopsia muestran que el TEP se mantiene cerca del 15\% como contribuyente o causa directa de muerte en pacientes hospitalizados en las últimas décadas ${ }^{2-6}$.

En los casos fatales, aproximadamente $2 / 3$ de los pacientes mueren durante la primera hora de presentación, en la mayoría de ellos como consecuencia de una embolia anatómicamente masiva o a émbolos más pequeños, submasivos o recurrentes ${ }^{6,7-9}$.

A pesar de que tradicionalmente se atribuye la letalidad del TEP al tamaño del émbolo, es fundamental considerar la reserva cardiopulmonar ${ }^{10}$. Al integrar el tamaño del émbolo y la función cardiopulmonar en el pronóstico del enfermo, el término de TEP severo o grave define cualquier combinación de éstos que determine un evento hemodinámicamente significativo (Figura 1).

La presencia de shock permite identificar rápidamente al subgrupo de pacientes con elevada mortalidad. Sin embargo, se desconoce la combinación exacta entre el tamaño del émbolo y la reserva cardiopulmonar que produce la inflexión en la curva de mortalidad (antes del shock clínicamente evidente). Reconocer este subgrupo de pacientes con macrohemodinamia estable, pero con un mayor riesgo de complicaciones y mortalidad, permitiría establecer un umbral por sobre el cual justificar un manejo más agresivo, como la trombolisis.

\section{Fisiopatología}

En el TEP masivo, el o los émbolos que impactan la vasculatura pulmonar generan un aumento en la resistencia o postcarga sobre el ventrículo 


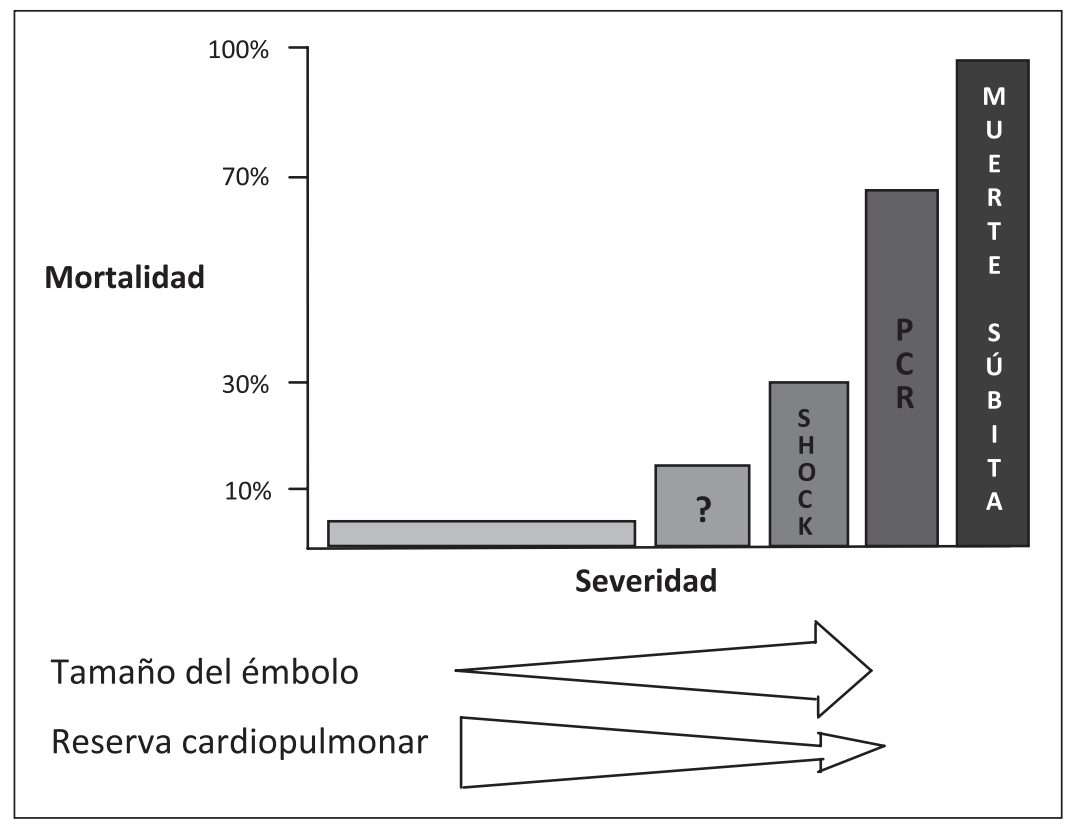

Figura 1. Relación entre severidad y mortalidad en pacientes con TEP. PCR: Paro Cardio-Respiratorio. derecho (VD). La magnitud de ésta se relaciona tanto con la obstrucción mecánica como con la reserva cardiopulmonar ${ }^{11-13}$.

Inicialmente, este aumento en la postcarga del VD es compensada por taquicardia (mediada por catecolaminas) y la reserva de precarga de la ley de Frank-Starling. Esto aumenta la presión y el radio en cavidades derechas y por ende la tensión parietal, principal determinante del consumo de oxígeno.

La perfusión coronaria del VD depende de la gradiente entre la presión arterial media (PAM) y la presión subendocárdica del VD. Así, la caída en la PAM sumada al aumento en la presión de fin de diástole del VD genera una disminución de la presión de perfusión y, por ende, del aporte de oxígeno. Se produce entonces, una situación de alta demanda de oxígeno (alta tensión parietal) y escaso flujo coronario que determina isquemia e insuficiencia del $\mathrm{VD}^{14}$.

Al caer la función sistólica del VD disminuye la precarga del ventrículo izquierdo (VI). Además, el desplazamiento del septum interventricular hacia la izquierda y la restricción pericárdica (ambos determinados por la dilatación del VD) contribuyen a disminuir aun más dicha precarga ${ }^{15-17}$.

Una vez sobrepasados los mecanismos de compensación tras la caída del volumen sistólico del VD, aparece hipotensión sistémica.
Es importante considerar también la posibilidad de recurrencias tromboembólicas precoces que agraven el cuadro y aceleren el colapso hemodinámico de un paciente previamente estable. $\mathrm{Al}$ respecto, hay estudios que demuestran mayor mortalidad en pacientes con TEP cuando existe trombosis venosa profunda (TVP) concomitan$\mathrm{te}^{18,19}$.

\section{Estratificando el riesgo}

El TEP representa un espectro de síndromes clínicos con implicancias pronósticas muy distintas. En este sentido, podemos agrupar a los pacientes en 3 grupos:

1. Pacientes con presión arterial normal y función del VD normal: Estos pacientes tienen excelente pronóstico sólo con tratamiento anticoagulante.

2. TEP masivo: Generalmente definido por la presencia de compromiso hemodinámico: shock o hipotensión (presión sistólica menor a $90 \mathrm{mmHg}$ ). A diferencia del grupo anterior, estos pacientes tienen un elevado riesgo de muerte y por ende, son manejados en forma agresiva (trombolisis).

3. TEP submasivo: Corresponde a aquellos pacientes con presión arterial normal pero con evidencias de disfunción ventricular derecha. Este grupo tiene mayor riesgo de morbimortalidad respecto a aquellos sin disfunción del VD 
y constituye un foco de controversia respecto al enfoque diagnóstico-terapéutico ideal.

La identificación de pacientes con TEP masivo es eminentemente clínica. La pesquisa de disfunción ventricular derecha en pacientes hemodinámicamente estables constituye un desafío para el clínico en el abordaje inicial de estos enfermos.

Existen distintas herramientas que permiten estimar el riesgo de mortalidad a corto plazo y la presencia de disfunción del VD:

\section{Clínica}

Al margen del shock como predictor de morbimortalidad, se han desarrollado distintos modelos pronósticos utilizando algunas variables clínicas. La mayoría de estos modelos tienen importantes limitaciones prácticas, permiten más bien identificar pacientes de bajo riesgo para manejo ambulatorio y no son utilizados con frecuencia ${ }^{20}$. El score simplificado PESI (Pulmonary Embolism Severity Index) está compuesto de 6 variables (suman 1 punto cada una): 1) Edad mayor a 80 años; 2) Antecedentes de cáncer; 3) Historia de enfermedad cardiopulmonar crónica; 4) Frecuencia cardiaca (FC) $>110 x^{\prime}$; 5) Presión arterial sistólica $<100 \mathrm{mmHg}$ y 6) Saturación de oxígeno $<90 \%$. Pacientes con score de 0 son catalogados de bajo riesgo, mientras pacientes con score de 1 o más como de alto riesgo. El valor predictivo positivo y negativo (VPP y VPN) del score para mortalidad a 30 días fue de 10,9 y $99 \%$ respectivamente, en el estudio de Jiménez et $\mathrm{al}^{21}$. Por ende, sirve para identificar con certeza pacientes de bajo riesgo susceptibles de tratamiento convencional, eventualmente ambulatorio sin tener que recurrir a otros estudios de imagen o biomarcadores.

\section{Electrocardiograma (ECG)}

Los hallazgos que sugieren sobrecarga ventricular derecha son: bloqueo de rama derecha, ondas T negativas en derivaciones V1 a V4 y el patrón "S1Q3T3" (S profunda en D1, Q en D3 y $T$ negativa en D3). En el registro MAPPET, aquellos pacientes que presentaban al menos una alteración electrocardiográfica (arritmias auriculares, bloqueo completo de rama derecha, ondas Q en D3 y aVF y alteraciones del segmento ST en derivaciones precordiales) tenían una mortalidad hospitalaria de $29 \%$ comparada con sólo $11 \%$ de aquellos que no presentaban anormalidades (OR $2,56, \mathrm{p}<0,001)^{22}$. En el estudio de Punukollu et al, la presencia de ondas $\mathrm{T}$ invertidas en derivaciones
V1 a V3 fue el hallazgo de mejor rendimiento para identificar disfunción ventricular derecha con una sensibilidad de $75 \%$ y una especificidad de $88 \%$, VPP y VPN de 86 y $78 \%$, respectivamente ${ }^{23}$. Así, el ECG puede constituir una herramienta de screening inicial de bajo costo y ampliamente disponible.

\section{Ecocardiograma}

Si bien no existen criterios estandarizados para definir la disfunción del VD, el ecocardiograma es probablemente la mejor imagen para evaluarla. Los hallazgos más relevante en este sentido son: 1) Hipoquinesia y dilatación del VD;2) Aplanamiento o movimiento paradojal del septum interventricular; 3) Regurgitación tricuspídea; 4) Hipertensión pulmonar determinada por velocidad del jet de regurgitación mayor a 2,6 m/seg; 5) Pérdida de colapso de la vena cava inferior ${ }^{24} y$ 6) Relación del diámetro final de diástole del VD/ VI mayor a 0,925. En la revisión sistemática de Sánchez et al, el riesgo relativo (RR) de mortalidad para pacientes con disfunción ventricular derecha ecocardiográfica fue 2,53 veces mayor que la de aquellos sin criterios de disfunción del VD. El VPP, sin embargo, fue sólo de $58 \%{ }^{26}$.

\section{Tomografía axial computada (TAC) de tórax}

Una relación entre los diámetros del VD/ VI mayor a 0,9 se ha asociado a mayor mortalidad $(27,28)$, sin embargo, la información sigue siendo poco concluyente. En el metaanálisis de Coutance et $\mathrm{a}^{29}$ que incluyó a 503 pacientes, el riesgo de mortalidad no fue significativamente mayor en pacientes con evidencias de disfunción ventricular derecha en el TAC (OR 1,54, IC 95\% $0,7$ a 3,4$)$. Utilizar este tipo de información tiene la ventaja de estratificar el riesgo con datos adquiridos en la imagen diagnóstica inicial.

\section{Biomarcadores}

Niveles elevados de troponina y péptido natriurético cerebral (conocido por su traducción al inglés como BNP: Brain Natriuretic Peptide) han demostrado asociación con mayor mortalidad. El BNP es una hormona liberada en respuesta al estiramiento de los miocitos. Es sintetizada como prohormona inactiva (pro-BNP) que luego se divide en la hormona activa (BNP) y el fragmento terminal inactivo (NT-pro-BNP). En el metaanálisis de Klok et al. los pacientes con elevaciones de BNP y NT-pro-BNP tenían un riesgo de mortalidad 6,5 
y 8,7 veces mayor que los pacientes con niveles normales respectivamente ${ }^{30}$. Sin embargo, el VPP para mortalidad es bajo, fluctuando en la mayoría de los estudios entre 10 y $20 \%$. Esto genera dudas al momento de utilizar este marcador en forma aislada como herramienta para definir una terapia más agresiva. Este fenómeno probablemente se deba a que la elevación del BNP ocurre en otras condiciones, como insuficiencia renal, enfermedades pulmonares crónicas, insuficiencia cardiaca y edad avanzada ${ }^{31}$. Si lo vemos de forma inversa, el VPN es extremadamente alto (97-99\%), por lo que cuando un paciente presenta niveles bajos de BNP o NT-pro-BNP, el pronóstico es excelente y su evolución será probablemente satisfactoria sólo con tratamiento anticoagulante ${ }^{32-35}$. Es importante destacar además que la mayoría de los estudios no estableció un punto de corte predefinido sino que éste fue determinado en forma retrospectiva mediante la construcción de curvas ROC. También hay que considerar que los niveles de BNP tardan varias horas en elevarse después de un evento agudo, lo que puede generar falsos negativos en pacientes que consultan precozmente ${ }^{36}$. Existen también diversos estudios que demuestran la utilidad de medir niveles de troponinas en la estratificación del riesgo de pacientes normotensos con TEP. En el metaanálisis más reciente que incluyó a 1366 pacientes normotensos con TEP sintomático, niveles elevados de troponina $\mathrm{T}$ o I se asociaron a un riesgo 4,26 veces mayor de mortalidad (IC 95\% $2,13$ a 8,5$)^{37}$. Sin embargo, los likelihood ratios (LR) no fueron lo suficientemente negativos o positivos para determinar cambios de conducta por sí solos (LR negativo para mortalidad de 0,59 y positivo de $2,26)$. Una limitación importante es que los estudios utilizan distintos puntos de corte para definir troponina anormal. Los autores concluyeron que el uso combinado de troponinas y otros exámenes que evidencien disfunción ventricular derecha, como el ecocardiograma o la TAC, pudiera ser una estrategia que mejore la capacidad de predecir un peor pronóstico y justificar tratamientos más agresivos. En una reciente publicación de nuestro grupo, estudiamos los cambios de biomarcadores cardiacos en pacientes con embolia pulmonar submasiva $^{38}$. Los niveles plasmáticos de NT-pro-BNP se correlacionaron directamente con disfunción del VD. Más importante, los pacientes experimentaron una reducción significativa tanto en los niveles de este marcador como en los de presión arterial pulmonar después de una trombolisis invasiva exitosa. Así, las mediciones seriadas de este biomarcador pudieran ser útiles como una herramienta de seguimiento para evaluar el éxito o el fracaso del tratamiento de la embolia pulmonar severa.

\section{TVP concomitante}

Como ya se mencionó, recurrencias precoces pueden agravar cuadros estables y producir colapso hemodinámico. En un estudio de cohorte prospectiva de 707 pacientes con diagnóstico de TEP, $51,2 \%$ presentó TVP concomitante. Estos pacientes tuvieron mayor riesgo de mortalidad global, 15,2 versus 6,4\% (HR 2,05, IC 95\% 1,24 a 3,38 ) y mortalidad asociada a TEP (HR 4,25, IC 95\% 1,61 a 11,25) en los 3 meses de seguimiento ${ }^{18}$. En nuestra casuística de 893 pacientes sometidos a angiografía pulmonar por TAC (angioTAC) y venografía por TAC por sospecha clínica de TEP, el $16 \%$ presentaba repercusión ventricular derecha en la tomografía. De ellos, $68,5 \%$ tenía un foco embolígeno concomitante $\mathrm{y}$, por ende, riesgo de una nueva embolia ${ }^{39}$.

\section{Dímero D}

Distintos estudios han demostrado que pacientes con dímero $\mathrm{D}$ elevado tienen trombos más extensos y de localización más proximal ${ }^{40-42}$. Hallazgos del registro internacional multicéntrico RIETE sugieren que también puede ser un marcador de riesgo de mortalidad precoz. En una población de 1.707 pacientes, la mortalidad a 15 días aumentaba en conjunto con los valores de dímero D. En el primer cuartil la mortalidad fue de $2,7 \%$, comparado con $7,0 \%$ en el cuarto cuartil (OR 1,8, IC 95\% 1,1 a 3,2). El VPP para mortalidad fue nuevamente bajo, sólo $7 \%{ }^{43}$.

Es claro entonces que la presencia de disfunción ventricular derecha en imágenes (Eco y TAC) o biomarcadores aumenta significativamente el riesgo de mortalidad. Sin embargo, desde un punto de vista clínico, el bajo VPP de estos test genera incertidumbre al momento de decidir en base a sus resultados, una conducta terapéutica con riesgos potencialmente letales, como la hemorragia intracerebral (HIC).

Surge la posibilidad de utilizar estos hallazgos en conjunto para definir con mayor claridad cuál paciente requiere una monitorización más estricta y/o trombolisis. Si bien en algunos estudios la 
combinación de ecocardiograma y biomarcadores no aumentó el rendimiento de cada test por separado $^{44}$, en su mayoría el uso combinado mejoró el VPP de mortalidad asociada a TEP ${ }^{45-47}$. El estudio de Jiménez et al ${ }^{48}$ evaluó la utilidad de 3 test diagnósticos (troponina I, ecocardiograma y ecografía Doppler de extremidades inferiores) para predecir el riesgo de mortalidad asociada a TEP a 30 días del diagnóstico. En dicho estudio, la combinación de ecocardiografía (o troponina si la Eco no estaba rápidamente disponible) y ecografía Doppler de extremidades inferiores, obtuvo el mejor rendimiento en términos de especificidad y VPP (mortalidad de $19,6 \%$, especificidad de $91,3 \%$ y VPP de $25 \%)$. El uso de los 3 test en forma simultánea no mejoró significativamente el rendimiento.

\section{Controversias en el tratamiento del TEP submasivo}

Se ha discutido ampliamente el rol potencial de tratamientos agresivos, como la trombolisis, en el subgrupo de pacientes con presión arterial normal y evidencias de disfunción ventricular derecha ${ }^{49-53}$.

En el mayor estudio aleatorio realizado (MAPPET-3), 256 pacientes con TEP submasivo (definido como evidencia ecocardiográfica de disfunción ventricular derecha, hipertensión pulmonar precapilar en un sondeo derecho o nuevos signos electrocardiográficos de sobrecarga derecha) fueron distribuidos en forma aleatoria a trombolisis con alteplasa $100 \mathrm{mg}$ iv en $2 \mathrm{~h}$, seguido de una infusión de heparina o a placebo más infusión de heparina ${ }^{54}$. El grupo sometido a trombolisis presentó una reducción significativa del outcome primario compuesto por muerte intrahospitalaria y deterioro clínico que requiera escalar en el tratamiento ( 11 vs $24,6 \%$, p: 0,006 ). Sin embargo, el diseño de dicho estudio permitía la apertura del protocolo ciego frente a cualquier deterioro considerado significativo por el tratante. No es extraño que la diferencia observada fuera casi completamente atribuible a un mayor número de trombolisis secundarias en el grupo asignado inicialmente a placebo.

El metaanálisis de Tardy et al incluyó a 464 pacientes con hemodinamia estable, distribuidos al azar para recibir alteplasa versus heparina ${ }^{55}$. Se observó una reducción no significativa en la mortalidad asociada a TEP o recurrencia en pacientes distribuidos al azar a recibir alteplasa (3,5 versus $4,6 \%$, IC $95 \% 0,38$ a 2,51). Los resultados fueron similares al analizar el subgrupo de pacientes con evidencia ecográfica de disfunción ventricular derecha.

Las últimas guías de la American Heart Association publicadas este año, señalan que la fibrinolisis puede ser considerada en pacientes con TEP submasivo con evidencia clínica de mal pronóstico (inestabilidad hemodinámica, insuficiencia respiratoria creciente, disfunción VD severa o necrosis miocárdica mayor) y bajo riesgo de complicaciones hemorrágicas (Clase IIb, Nivel de evidencia $\mathrm{C})^{56}$.

Si revisamos los datos expuestos, la mortalidad a corto plazo de los pacientes con TEP submasivo tratados con anticoagulación no es mayor al 3-5\%. Así, aunque la fibrinolisis genere una reducción relativa importante en la mortalidad (por ejemplo 30-50\%), el efecto neto probablemente no supere el 1-2\%. Es importante mirar otros resultados como hipertensión pulmonar crónica y calidad de vida en pacientes con TEP submasivo que han o no sido sometidos a trombolisis y las potenciales complicaciones del tratamiento trombolítico, en particular la hemorragia intracerebral (HIC).

En relación a la hipertensión pulmonar, el estudio de Kline et al, enroló a 200 pacientes normotensos, con TEP demostrado por TAC y Ecocardiograma para estimar presión sistólica de arteria pulmonar (PSAP); 162 de los 180 sobrevivientes fueron evaluados 6 meses después del evento (144 tratados con heparina y 18 con heparina más alteplasa). 27\% de los pacientes no trombolizados presentó una PSAP a los 6 meses mayor que al momento del diagnóstico. Esto se asoció a una menor capacidad funcional e intolerancia al ejercicio. En el grupo trombolizado, la PSAP no fue mayor al momento del control, en ninguno de los pacientes ${ }^{57}$.

Respecto a las complicaciones hemorrágicas, las mayores alcanzan al 13\% (la mayoría de ellas asociadas a sangrado en el sitio de punción de la angiografía pulmonar en estudios antiguos) y las HIC o hemorragias fatales $1,8 \%{ }^{58}$. La edad sobre 70 años ha demostrado ser un factor de riesgo importante para HIC, no habiéndose descrito prácticamente casos bajo los 50 años $^{59,60}$.

El estudio PEITHO (NCT00639743) iniciado el año 2009 espera reclutar 1.000 pacientes con TEP y evidencias ecocardiográficas de sobrecarga 
ventricular derecha asociado a niveles elevados de troponina I o T. Los pacientes serán distribuidos al azar a recibir trombolisis con tenecteplasa o placebo. Los resultados de este estudio se esperan para el año 2013 y debiesen aclarar el rol de la trombolisis en este subgrupo especial de pacientes.

\section{Conclusiones}

Existe un subgrupo de enfermos con macrohemodinamia normal pero con disfunción ventricular derecha, lo que les confiere un mayor riesgo de morbimortalidad.

La presencia de variables clínicas como shock o hipotensión, por un lado, y la ausencia de otras como aquellas especificadas en el score sPESI, permiten identificar pacientes con TEP de muy alto o muy bajo riesgo respectivamente y orientar el tratamiento en forma concordante ${ }^{61}$.

El uso de imágenes y biomarcadores podría ayudar a identificar a un subgrupo de enfermos con mayor riesgo que justifique una monitorización estricta y un manejo más agresivo (trombolisis). El bajo valor predictivo positivo de cada uno de estos exámenes no ha permitido aun identificar con claridad cuales pacientes normotensos pudieran beneficiarse de este tipo de terapia. Por otra parte, su uso combinado parece mejorar nuestra capacidad de identificar a estos pacientes.

La decisión de ofrecer o no tratamiento trombolítico a pacientes con TEP submasivo debe basarse no sólo en el análisis de los potenciales beneficios, sino también en el de las complicaciones hemorrágicas.

\section{Referencias}

1. Piazza G, Goldhaber SZ. Management of Submassive Pulmonary Embolism. Circulation 2010; 122: 1124-9.

2. Nordstrom M, Lindblad B. Autopsy-verified venous thromboembolism within a defined urban population: the city of Malmo, Sweden. APMIS 1998: 106: 378-84.

3. Coon WW, Coller FA. Clinicopathologic correlation in thromboembolism. Surg Gynecol Obstet 1959: 109: 259-69.

4. Morrell MT, Dunnill MS. The post-mortem incidence of major pulmonary embolism. Br J Surg 1968; 55: 347-52.

5. Stein PD, Henry Jw. Prevalence of acute pulmonary embolism among patients in a general hospital and at autopsy. Chest 1995; 108: 978-81.
6. Twigg SJ, McCrirrick A, Sanderson PM. A comparison of post mortem findings with post hoc estimated clinical diagnoses of patients who die in a United Kingdom intensive care unit. Intensive Care Med 2001; 27: 70610.

7. Tibbut DA, Davies JA, Anderson JA. Comparison by controlled clinical trial of streptokinase and heparin in treatment of life-threatening pulmonary embolism. BMJ 1974; 1:343-7.

8. Morrison MCT. Is pulmonary embolectomy obsolete? Br J Dis Chest 1963; 57: 187-91.

9. Gifford RW Jr, Goves Lk. Limitations in the feasibility of pulmonary embolectomy: a clinicopathologic study of 101 cases of massive pulmonary embolism. Circulation 1969; 39: 523-30.

10. Wood KE. Major Pulmonary Embolism, Review of a Pathophysiologic Approach to the Golden Hour of Hemodynamically Significant Pulmonary Embolism. Chest 2002; 121: 877-905.

11. Dalen JE, Haynes FW, Hoppin FG. Cardiovascular responses to experimental pulmonary embolism. Am J Cardiol 1967; 20: 3-9.

12. McIntrye KM, Sasahara AA. Hemodynamic and ventricular responses to pulmonary embolism. Prog Cardiovasc Dis 1974; 17: 175-90.

13. Parker BM, Smith Jr. Pulmonary embolism and infraction: a review of the physiologic consequences of pulmonary artery obstruction. Am J Med 1958; 24: 402-27.

14. Vlahakes GJ, Turley K, Hoffman JI. The pathophysiology of failure in acute right ventricular hypertension: hemodynamic and biochemical correlations. Ciruclation 1981; 63: 87-95.

15. Taylor RR, Covell JW, Sonnenblick EH. Dependence of ventricular distensibility on filling of the opposite ventricle. Am J Physiol 1967; 213: 711-8.

16. Jardin F, Dubourg O, Gueret P. Quantitative twodimensional echocardiography in massive pulmonary embolism: emphasis on ventricular interdependence and leftward septal displacement. J Am Coll Cardiol 1987; 10: 1201-6.

17. Belenkie I, Dani R, Smith ER. Ventricular interaction during experimental acute pulmonary embolism. Circulation 1988; 78: 761-8.

18. Jiménez D, Aujesky D, Díaz G, Monreal M, Otero R, Martí D, et al. Prognostic Significance of Deep Vein Thrombosis in Patients Presenting with Acute Symptomatic Pulmonary Embolism. Am J Resp Crit Care Med 2010; 181: 983.991.

19. Yamaki T, Nozaki M, Sakura H, Takeuchi M, Soejima K, Kono T. Presence of lower limb deep vein thrombosis and prognosis in patients with symptomatic pulmonary 
embolism: preliminary report. Eur J Vasc Endovasc Surg 2009; 37: 225-31.

20. Jiménez D, Yusen RD. Prognostic models for selecting patients with acute pulmonary embolism for initial outpatient therapy. Curr Opin Pulm Med 2008; 14: 414-21.

21. Jiménez D, Aujesky D, Moores L, Gómez V, Lobo JL, Uresandi F, et al. Simplification of the Pulmonary Embolism Severity Index for Prognostication in Patients With Acute Symptomatic Pulmonary Embolism. Arch Intern Med 2010; 170: 1383-9.

22. Geibel A, Zehender M, Kasper W, Olschewski M, Klima C, Konstantinides SV. Prognostic value of the ECG on admission in patients with acute major pulmonary embolism. Eur Respir J 2005; 25: 843-8.

23. Punukollu G, Gowda RM, Vasavada BC, Khan IA. Role of Electrocardiography in Identifying Right Ventricular Dysfunction in Acute Pulmonary Embolism. Am J Cardiol 2005; 96: 450-2.

24. Goldhaber SZ. Echocardiography in the management of pulmonary embolism. Ann Intern Med 2002; 136: 691-700.

25. Fremont B, Pacouret G, Jacobi D, Puglisi R, Charbonnier B, de Labriolle A. Prognostic value of echocardiographic right/left ventricular end-diastolic diameter ratio in patients with acute pulmonary embolism: results from a monocenter registry of 1416 patients. Chest 2008; 133 : 358-62.

26. Sánchez O, Trinquart L, Colombet I, Durieux P, Huisman MV, Chatellier G, et al. Prognostic value of right ventricular dysfunction in patients with haemodynamically stable pulmonary embolism: a systematic review. Eur Heart J 2008; 29: 1569-77.

27. Schoepf UJ, Kucher N, Kipfmueller F, Quiroz R, Costello P, Goldhaber SZ. Right ventricular enlargement on chest computed tomography: a predictor of early death in acute pulmonary embolism. Circulation 2004; 110: 3276-80.

28. van der Meer RW, Pattynama PM, van Strijen MJ, van den Berg-Huijsmans AA, Hartmann IJ, Putter H, et al. Right ventricular dysfunction and pulmonary obstruction index at helical CT: prediction of clinical outcome during 3-month follow-up in patients with acute pulmonary embolism. Radiology 2005; 235: 798-803.

29. Coutance G, Cauderlier E, Ehtisham J, Hamon M, Hamon M. The prognostic value of markers of right ventricular dysfunction in pulmonary embolism: a meta-analysis. Critical Care 2011: 15; R103.

30. Klok FA, Mos ICM, Huisman MV. Brain-Type Natriuretic Peptide Levels in the Prediction of Adverse Outcome in Patients with Pulmonary Embolism. Am J Respir Crit Care Med 2008; 178: 425-30.

31. de Lemos JA, McGuire DK, Drazner MH. B-type natriu- retic peptide in cardiovascular disease. Lancet 2003; 362: 316-22.

32. Kucher N, Printzen G, Doernhoefer T, Windecker S, Meier B, Hess OM. Low pro-brain natriuretic peptide levels predict benign clinical outcome in acute pulmonary embolism. Circulation 2003; 107: 1576-8.

33. Kucher N, Printzen G, Goldhaber SZ. Prognostic role of brain natriuretic peptide in acute pulmonary embolism. Circulation 2003; 107: 2545-7.

34. Ten Wolde M, Tulevski II, Mulder JW, Sohne M, Boomsma F, Mulder BJ, et al. Brain natriuretic peptide as a predictor of adverse outcome in patients with pulmonary embolism. Circulation 2003; 107: 2082-4.

35. Pruszczyk P, Kostrubiec M, Bochowicz A, Styczynski G, Szulc M, Kurzyna M, et al. N-terminal pro-brain natriuretic peptide in patients with acute pulmonary embolism. Eur Respir J. 2003; 22: 649-53.

36. Hama N, Itoh $\mathrm{H}$, Shirakami G, Nakagawa O, Suga S, Ogawa Y, et al. Rapid ventricular induction of brain natriuretic peptide gene expression in experimental acute myocardial infarction. Circulation 1995; 92: 1558-64.

37. Jiménez D, Uresandi F, Otero R, Lobo JL, Monreal M, Martí D, et al. Troponin-Based Risk Stratification of Patients With Acute Nonmassive Pulmonary Embolism. CHEST 2009; 136: 974-82.

38. Andresen M, González A Mercado M, Díaz O, Meneses If Fava M et al. Natriuretic peptide type-B can be a marker of reperfusion in patients with pulmonary thromboembolism subjected to invasive treatment. Int J Cardiovasc Imaging 2011 Apr 12 [Epub ahead of print].

39. Andresen M, González A, Díaz O, Meneses L, Fava M, Orlandini E, et al. Evaluación prospectiva de la venografía mediante angioTC en el diagnóstico de enfermedad tromboembólica. Rev Med Chile 2010; 138: 1480-6.

40. Galle C, Papazyan JP, Miron MJ, Solsman D, Bounameaux H, Perrier A. Prediction of pulmonary embolism extent by clinical findings, $\mathrm{D}$-dimer level and deep vein thrombosis shown by ultrasound. Thromb Haemost 2001; 86: 1156-60.

41. Ghanima W, Abdelnoor M, Holmen LO, Nielssen BE, Ross S, Sandset PM. D-dimer level is associated with the extent of pulmonary embolism. Thromb Res 2007; 120: 281-8.

42. Hochuli M, Duewell S, Frauchiger B. Quantitative ddimer levels and the extent of venous thromboembolism in CT angiography and lower limb ultrasonography. Vasa 2007; 36: 267-74

43. Lobo JL, Zorrilla V, Aizpuru F, Grau E, Jiménez D, Palareti G, Monreal M and The Riete Investigators. Ddimer levels and 15-day outcome in acute pulmonary 
emoblism. Findings from the RIETE Registry. J Thromb Haemost 2009; 7: 1795-801.

44. Kostrubiec M, Pruszczyk P, Bochowicz A, Pacho R, Szulc M, Kaczynska A, et al. Biomarker-based risk assessment model in acute pulmonary embolism. Eur Heart J 2005; 26: 2166-72.

45. Binder L, Pieske B, Olschewski M, Geibel A, Klostermann B, Reiner C, et al. N-terminal pro-brain natriuretic peptide or troponin testing followed by echocardiography for risk stratification of acute pulmonary embolism. Circulation 2005; 112: 1573-9.

46. Scridon T, Scridon C, Skali H, Álvarez A, Goldhaber SZ, Solomon SD. Prognostic significance of troponin elevation and right ventricular enlargement in acute pulmonary embolism. Am J Cardiol 2005; 96: 303-5.

47. Stein PD, Matta F, Janjua M, Yaekoub AY, Jaweesh F, Alrifai A. Outcome in stable patients with acute pulmonary embolism who had right ventricular enlargement and/or elevated levels of troponin I. Am J Cardiol 2010; 106: 558-63.

48. Jiménez D, Aujesky D, Moores L, Gómez V, Martí D, Briongos S, et al. Combinations of prognostic tolos for identification of high-risk normotensive patients with acute symptomatic pulmonary embolism. Thorax 2011; 66: 75-81.

49. Thabut G, Logeart D. Thrombolysis for Pulmonary Embolism in Patients With Right Ventricular Dysfunction. Con. Arch Intern Med 2005; 165: 2200-3.

50. Proudfoot A, Melley D, Shah PL. Role of thrombolysis in haemodynamically stable patients with pulmonary embolism. Thorax 2008; 63: 853-4.

51. Perlroth DJ, Sanders GD, Gould MK. Effectiveness and Cost-effectiveness of Thrombolysis in Submassive Pulmonary Embolism. Arch Intern Med 2007; 167: 74-80.

52. Todd JL, Tapson FT. Thrombolytic Therapy for Acute Pulmonary Embolism. A Critical Appraisal. Chest 2009; 135: 1321-9.

53. Moores LK. Informing Decision Making for Patients With Acute Pulmonary Embolism. Chest 2009; 136: 952-3.
54. Konstantinides S, Geibel A, Heusel G, Heinrich F, Kasper W. Heparin plus Alteplase compared with Heparin alone in patients with submassive pulmonary embolism. N Engl J Med 2002; 347: 1143-50.

55. Tardy B, Venet C, Zeni F, Coudrot M, Guyomarc'h S, Mismetti P. Short term effect of recomibinant tissue plasminogen activator in patients with hemodynamically stable acute pulmonary embolism: Results of a meta-analysis involving 464 patients. Thromb Res 2009; 124: 672-7.

56. Jaff MR, McMurtry MS, Archer SL, Cushman M, Goldenberg N, Goldhaber SZ, et al. Management of Massive and Submassive Pulmonary Embolism, Iliofemoral deep Vein Thrombosis, and Chronic Thromboembolic Pulmonary Hypertension. Circulation 2011; 123: 0000 .

57. Kline JA, Steuerwald MT, Marchick MR, HernándezNino J, Rose GA. Prospective evaluation of right ventricular function and functional status 6 months after acute submassive pulmonary embolism: frequency of persistent or subsequent elevation in estimated pulmonary artery pressure. Chest 2009; 136: 1202-10.

58. Mareike L, Konstantinides S. Mortality Risk Assessment and the Role of Thrombolysis in Pulmonary Embolism. Crit Care Clin 2011; 27: 953-67.

59. Kanter DS, Mikkola KM, Patel SR, Parker JA, Goldhaber SZ. Thrombolytic Therapy for Pulmonary Emolism, Frequency of Intracraneal Hemorrhage and Associated Risk Factors. Chest 1997; 111: 1241-45.

60. Mikkola KM, Patel SR, Parke JA, Grodstein F, Goldhaber SZ. Increasing age is a major risk factor for hemorrhagic complications after pulmonary embolism thrombolysis. Am Heart J 1997; 134: 69-72.

61. Lankeit M, Gómez V, Wagner C, Aujesky D, Recio M, Briongos $\mathrm{S}$, et al. A strategy combining imaging and laboratory biomarkes in comparison to a simplified clinical score for risk stratification of patients with acute pulmonary embolism. Chest. Prepublished online August 18, 2011; DOI 10.1378/chest.11-1355. 